Document downloaded from:

http://hdl.handle.net/10251/168595

This paper must be cited as:

Samaniego-Riera, D.; Zoireff, G.; Vidal Rodriguez, B. (2021). Brillouin-Induced Dynamic Arbitrary Birefringence. Journal of Lightwave Technology. 39(7):1961-1967. https://doi.org/10.1109/JLT.2020.3044152

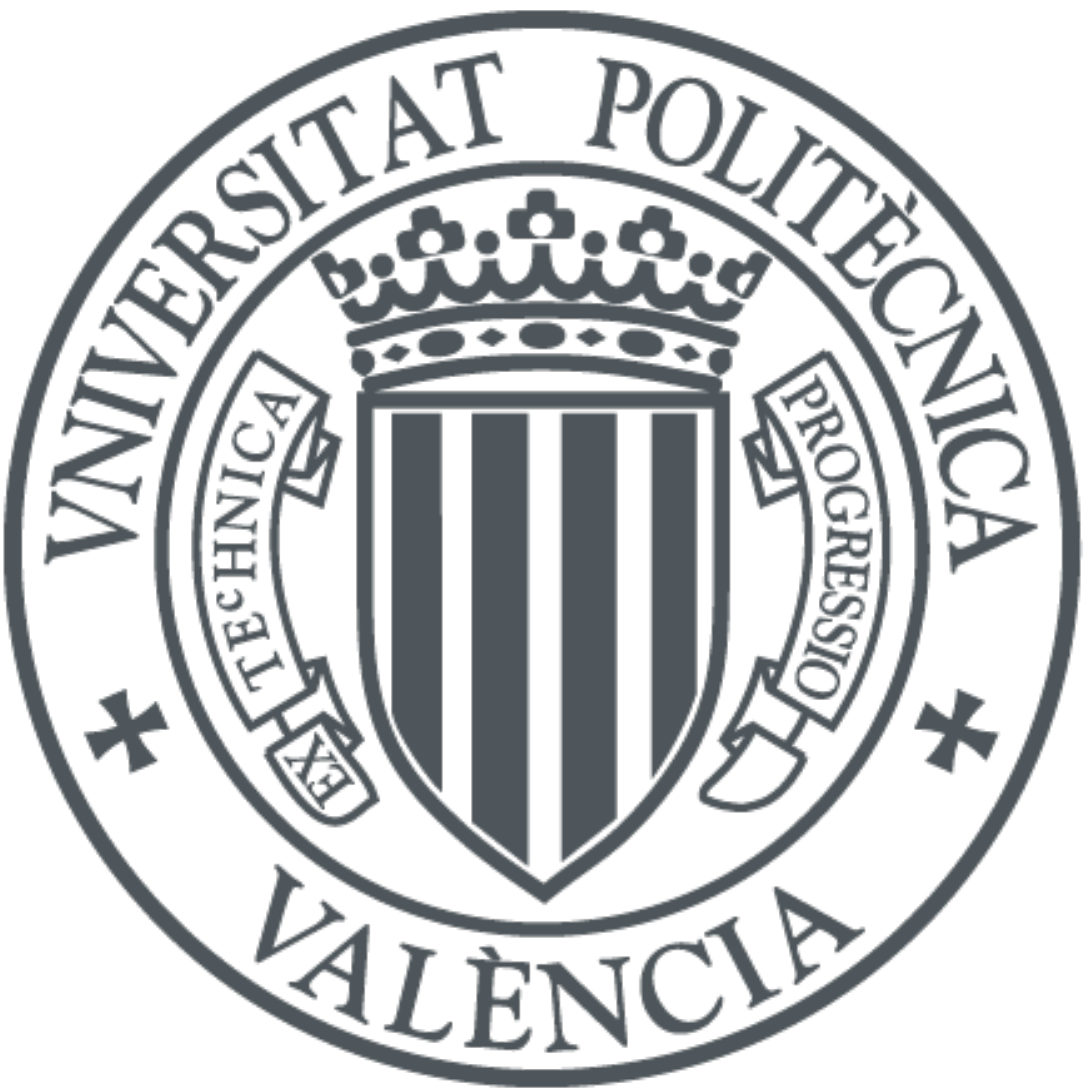

The final publication is available at

https://doi.org/10.1109/JLT.2020.3044152

Copyright Institute of Electrical and Electronics Engineers

Additional Information 


\title{
Brillouin-induced Dynamic Arbitrary Birefringence
}

\author{
D. Samaniego, G. Zoireff, and B. Vidal, Senior Member, IEEE
}

B irefringence is a fundamental property of matter that $i 61$ exploited in a wide range of optical devices, from 62

46

Abstract - A nonlinear method to generate and control both th $\$ 7$ type and magnitude of the birefringence, as well as differential 8 group delay (DGD) in optical fibers is studied. Experiments shoy 9 that both parameters of birefringence can be dynamically changed with only a slight variation of the SBS-induced gain of the system. The method is based on exploiting polarization dependence of 1 stimulated Brillouin scattering in optical fibers. The generation of 2 controlled DGD and DGD dispersion (DGDD) is als53 demonstrated. Proof-of-concept experiments are provide 4 showing the feasibility of dynamically inducing linear, circula 55 and elliptical birefringence, as well as DGD and DGDD.

Index Terms-Stimulated Brillouin Scattering, polarization, 57 birefringence, differential group delay, group velocity dispersion?

waveplates and liquid crystal displays to optical modulators an 63 specialty fibers. Natural anisotropic materials an $\$ 4$ metamaterials showing form birefringence exhibit a constan $\$ 5$ magnitude of this parameter for a given direction that can b86 linear, circular or, in general, elliptical. For example 67 waveplates for polarization control exhibit a constant amounf8 of birefringence and, thus, they are used to change in a fixed 8 way the state of polarization (SOP) of a signal.

Some materials allow the control of the magnitude of the 1 birefringence by applying an electric field that mainly induces a linear birefringence through Kerr effect or a magnetic field 2 which induces circular birefringence (optical activity) through Faraday effect. However, in these only the amount of 3 birefringence can be dynamically controlled.

Further versality could be achieved if not only the magnitude 5 of the birefringence but also its type could be dynamically 6 changed, for example, to go from linear to circular 7 birefringence. Artificially structured metamaterials [1-2] hav 98 been designed to offer arbitrary birefringence, which can offer 9 additional degrees of freedom in the design of optical systems [3]. Devices able to dynamically change its fundamental properties can be leveraged to enlarge the collection o $\$ 1$ reconfigurable optical systems in fields such as optica $\$ 2$ networks and sensing.

Nonlinear phenomena offer an alternative path to tackle thi\$4 challenge. Stimulated Brillouin scattering (SBS) [4] has 85 polarization-dependent behavior [5] and it has been used to implement polarization pulling [6] through its amplitude response as well as analogues to polarization controllers through its phase response [7]. In parallel, group delay control (slow light) using SBS has been extensively studied for optical buffering [8-12] but little attention has been paid to exploiting the polarization dependence of delays generated from SBS [13].

Here, we report on the use of a nonlinear effect to dynamically induce birefringence in optical fibers controlling both its amount and its type as well as differential group delay by exploiting the polarization dependence of SBS [5]. This method to achieve arbitrary birefringence can be applied to the synthesis of PMD emulators as well as a new tool in optical signal processing.

\section{THEORY AND OPERATING PRINCIPLE}

An isotropic transmission medium in which the refractive indexes of orthogonal propagation modes $\left(L P_{01 x}\right.$ and $L P_{01 y}$ in a single-mode optical fiber), $n_{x}$ and $n_{y}$, are different, is known as birefringent. Birefringence, $B_{m}$, is defined as

$$
B_{m}=\left|n_{x}-n_{y}\right|
$$

The change in the refractive index between orthogonal axes is related to the polarization-dependent phase shifts produced in the propagating medium according to

$$
\begin{aligned}
& n_{x}=n_{0}+\Delta n_{x}=n_{0}+\phi_{x} \frac{\lambda}{2 \pi L} \\
& n_{y}=n_{0}+\Delta n_{y}=n_{0}+\phi_{y} \frac{\lambda}{2 \pi L}
\end{aligned}
$$

where $L$ is the fiber length, $n_{0}$ is the effective refractive index, and $\phi_{x}, \phi_{y}$ are the polarization-dependent phase shifts generated in the optical fiber. Thus, birefringence can be manipulated via polarization-dependent phase shifts,

$$
B_{m}=\left|n_{x}-n_{y}\right|=\frac{\lambda}{2 \pi L}\left|\phi_{x}-\phi_{y}\right|
$$

SBS is a compelling candidate to dynamically induce such phase changes. Brillouin scattering originates from the inelastic scattering of photons by acoustic phonons in optical materials. Due to electrostriction in the optical waveguide, a moving
Submitted September $10^{\text {th }} 2020$.

D. Samaniego, G. Zoireff and B. Vidal are with the Nanophotonics Technology Center (NTC), Universitat Politècnica de València, Valencia,
46022, Spain (e-mail: bvidal@dcom.upv.es). G. Zoireff is also a fellow of CONICET and a member of the Laboratorio de Investigaciones Aplicadas en Telecomunicaciones (LIAT) (CNEA), Bariloche, Argentina. 
1 acoustic wave is generated which transfers energy from the 2 pump to a counterpropagating signal, creating a gain frequency 3 response. Thus, the pump coherently amplifies the optical probe 4 at the downshifted Stokes frequency. At the same time, the ${ }^{4}$ 5 counterpropagating signal transfers energy to the pump at the 2 6 upshifted anti-Stokes frequency, inducing a loss frequency 43 7 response. Both gain and loss processes also induce a phase shiff 44 8 as given by the Kramers-Kronig relations.

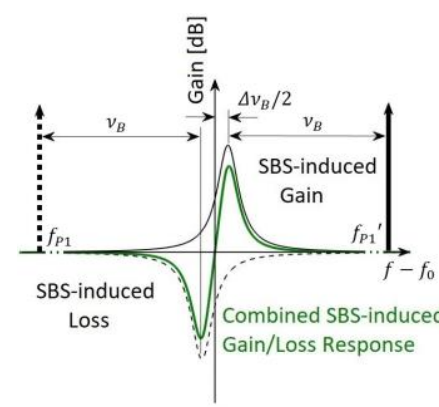

(a)

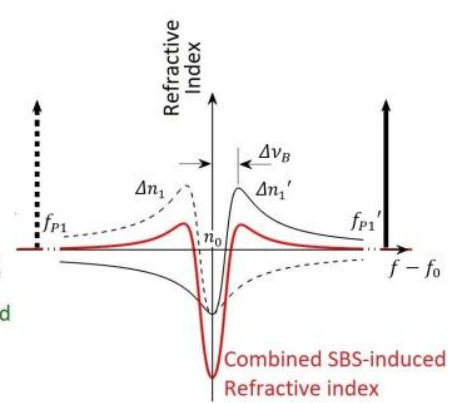

(b) where $g_{0}$ is the Brillouin gain factor, $P_{p}$ is the pump power, $L_{e f} 56$ is the effective interaction length of the fiber, $A_{\text {eff }}$ is th 98 effective area of the fiber [7].

59

If SBS is induced in single-mode fibers with fixed low birefringence, its gain and phase responses have polarization -60 dependent characteristics [4]. This polarization dependence of SBS is introduced by the $\xi$ factor, which is $2 / 3$ if th 61 counterpropagating signal SOP is aligned to the pump SOP, and 62 $1 / 3$ if they are orthogonal.

A gain plus a loss SBS response can be combined, as shown 63 in Fig. 1a, if a symmetric pair of pumps is used. Thus, the 65 combined amplitude response is always compensated whereas the refractive index reaches a negative minimum at th66 frequency of interest $f_{0}$, as shown in Fig $1 \mathrm{~b}$.

From the signal perspective, during its propagation through the fiber it experiences different refractive indexes according to its SOP. At the frequency of interest $f_{0}$, the signal effectively experiences the following variations around the effective refractive index $n_{0}$

Fig. 1. Combined SBS-induced (a) gain (green) and (b) refractive index (red) responses generated by two pumps symmetrically placed at $f_{0} \mp\left(v_{B}+\right.$ $\left.\Delta v_{B} / 2\right)$. Dashed lines and solid lines indicate the effects generated by $f_{P 1}$ and $f_{P 1}{ }^{\prime}$, respectively.

$$
\begin{aligned}
& \Delta n_{1 x}=-\xi_{\|} \frac{g_{0} P_{p} L_{e f f} c}{A_{e f f} 2 \pi L f_{0}} \\
& \Delta n_{1 y}=-\xi_{\perp} \frac{g_{0} P_{p} L_{e f f} c}{A_{e f f} 2 \pi L f_{0}} .
\end{aligned}
$$

When the SOP of the signal is aligned with the pump's SOP, i.e. the $x$ axis, it turns out that $\xi=\xi_{\|}=2 / 3$. On the other hand, when it is aligned to the $y$ axis (orthogonal SOP) $\xi=\xi_{\perp}=1 / 3$. This difference in both factors $\delta \xi=\xi_{\|}-\xi_{\perp}=1 / 3$ is sufficient to generate birefringence via SBS.

Now, let's consider a secondary pump pair, $f_{P 2}$ and $f_{P 2}{ }^{\prime}$, at $f_{0} \mp\left(v_{B}-\Delta v_{B} / 2\right)$ set along the $y$ polarization axis. Their SBS responses are similar to those in Fig. 1, but the induced refractive index variations reach a maximum positive value at $f_{0}$

$\mathrm{s}$

The variation in the refractive index induced by the primary and secondary pumps at the central frequency $f_{0}$ is

$$
\begin{aligned}
& \Delta n_{x 0}=\Delta n_{1 x}+\Delta n_{2 x}=-\delta \xi \frac{g_{0} P_{p} L_{e f f} c}{A_{e f f} 2 \pi L f_{0}} \\
& \Delta n_{y 0}=\Delta n_{1 y}+\Delta n_{2 y}=\delta \xi \frac{g_{0} P_{p} L_{e f f} c}{A_{e f f} 2 \pi L f_{0}} .
\end{aligned}
$$

Thus, the total birefringence, defined in (1), can be maximized in the band of interest
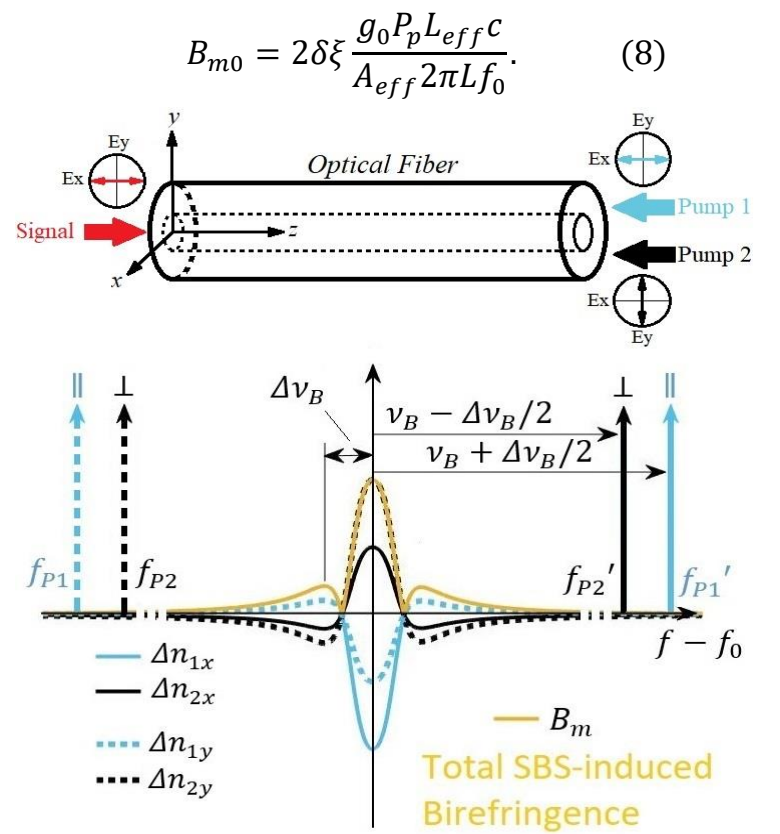

Fig. 2. Maximization of birefringence in an optical fiber at $f_{0}$ using primary $\left(f_{P 1}, f_{P 1}{ }^{\prime}\right)$ and secondary $\left(f_{P 2}, f_{P 2}{ }^{\prime}\right)$ pairs of pumps. The variation of refractive index induced along $x$ and $y$ axis are plot in solid and dashed lines, respectively. 


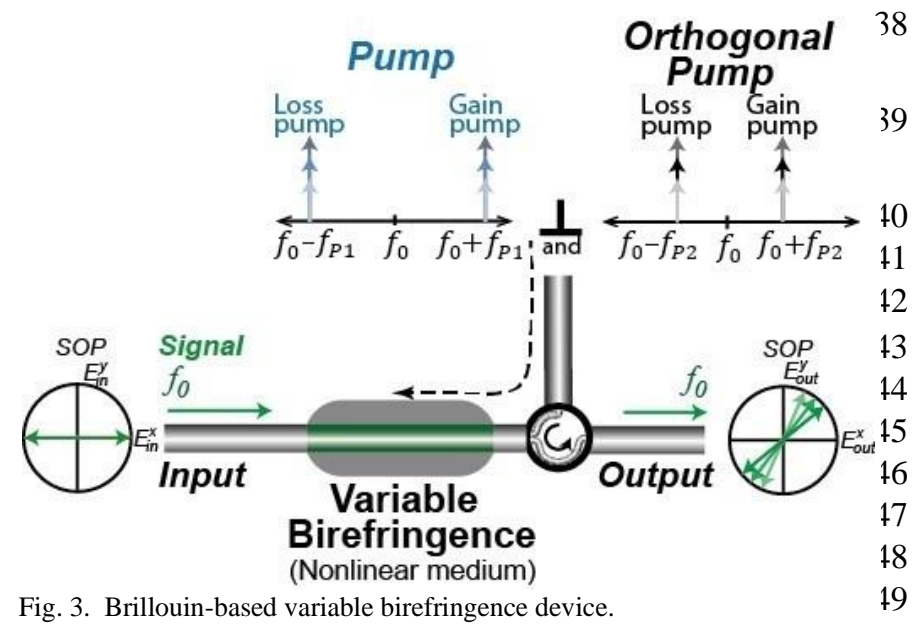

1

2

This process is illustrated in Fig. 2, using two pairs of orthogonal pumps to combine their responses to maximize the birefringence induced at $f_{0}$.

Each pair of pump waves can easily be achieved by biasing an external modulator at the minimum bias point (MITB) with a microwave signal of frequency, as shown in Fig. 3.

\section{A. SBS-induced Dynamic Arbitrary Birefringence}

The previous analysis has been carried out considering signal and pumps on an $x-y$ basis state. It can be extended to any orthogonal basis in order to arbitrarily manipulate the system birefringence.

The birefringence induced through SBS can be expresse 0 using the generalized Jones matrix formalism as,

$$
J=F^{-1} J_{S B S} F
$$

where $\boldsymbol{J}$ is the Jones matrix of the nonlinearly induced birefringence, and $\boldsymbol{F}$ is the transformation matrix related to the 55 arbitrary SOP of the pump. It can be calculated from the SOP6 of the orthogonal pumps as basis states [5], and $\boldsymbol{J}_{\boldsymbol{S} \boldsymbol{B} \boldsymbol{S}} \mathbf{5 7}$ Considering the $x-y$ basis state, $J_{S B S}$ is given by
$\boldsymbol{J}_{S B S}$

$$
=\left(\begin{array}{cc}
G_{1 \|} A_{1 \|} G_{2 \perp} A_{2 \perp} \cdot \exp \left(\mathrm{i} \frac{\pi L f_{0}}{c} B_{m 0}\right) & 0 \\
0 & G_{1 \perp} A_{1 \perp} G_{2 \|} A_{2 \|} \cdot \exp \left(-\mathrm{i} \frac{\pi L f_{0}}{c} B_{m 0}\right)
\end{array}\right)
$$

where $G_{\|}$and $G_{\perp}$ are the maximum and minimum Brillouip 6 gains, respectively, whilst $A_{\|}$and $A_{\perp}$ are the maximum an 87 minimum Brillouin loss, and $B_{m 0}$ is the maximum 8 birefringence generated at $f_{0}$ from both the primary an $\$ 9$ secondary pumps. When the gain and loss amplitude responses overlap, they compensate each other, i.e. $\left|G_{\|}\right| \cdot\left|A_{\|}\right| \cong 1$, and 0 $\left|G_{\perp}\right| \cdot\left|A_{\perp}\right| \cong 1$. Thus, the system behavior is equivalent to an isotropic retarder without significant power change at $f_{0}^{71}$ granting transparency to the nonlinear media.

The effect of the pump SOP on the induced birefringence is 3 given by the following transformation matrix

F

$$
=\left(\begin{array}{cc}
\cos \left(\delta+\frac{\pi}{4}\right) \exp \left(-j \varphi_{x}\right) & -\sin \left(\delta+\frac{\pi}{4}\right) \exp \left(j \varphi_{y}\right) \\
\sin \left(\delta+\frac{\pi}{4}\right) \exp \left(-j \varphi_{y}\right) & \cos \left(\delta+\frac{\pi}{4}\right) \exp \left(j \varphi_{x}\right)
\end{array}\right)
$$

where $\delta$ is the azimuth of the major axis of the ellipse of polarization of the pump and $\varphi_{x}, \varphi_{y}$ represent the phases of the electric-field components along $x$ and $y$ axes.

Linear birefringence is obtained when the pump pairs have linear SOPs (horizontal and vertical) as shown in Fig. 4a. For circular birefringence, pump pairs with left and right circular polarizations are used instead (Fig. 4b). In general, elliptical birefringence is obtained when the SOP of the pump pairs is elliptical (Fig. 4c).

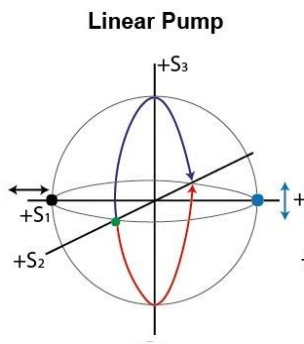

(a)

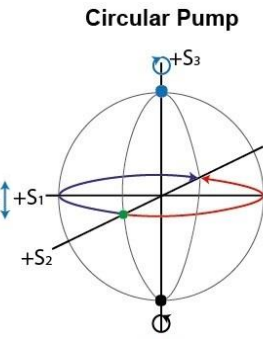

(b)

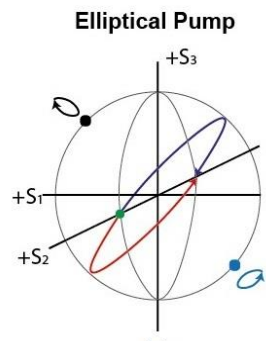

(c)
Fig. 4. Dependence of the type of birefringence with the SOP of the pump signal: (a) linear birefringence with linear pump (b) circular birefringence with circular pump and (c) elliptical birefringence with elliptical pump.

Thus, arbitrary types of transparent birefringent elements can be implemented by choosing the state of polarization of the pump. The amount of birefringence is determined by the pump power and the reconfigurability speed is mainly given by the length of the medium used for the Brillouin interaction.

\section{B. SBS-induced Dynamic Differential Group Delay}

The SBS-induced birefringence is associated to a variation in the group velocity of orthogonal polarization axes $\left(\tau_{g x}, \tau_{g y}\right)$, i.e. it could induce a differential group delay (DGD)

$$
\mathrm{DGD}=\left|\tau_{g x}-\tau_{g y}\right|=\frac{\omega}{c} \frac{d B_{m}}{d \omega}
$$

with $\omega$ the angular frequency and $c$ the speed of light.

As it can be seen in Fig. 2, if only the primary pumps, $f_{P 1}$ and $f_{P 1}{ }^{\prime}$, are applied, birefringence has a peak value at $f_{0}$, which corresponds to zero group delay at this frequency and consequently, no DGD.

On the other hand, maximum/minimum group delay along $x$ axis is located approximately at $f_{0} \pm \Delta v_{B} / 2$,

$$
\tau_{g x-\max / \min }=\tau_{g 1 \|}+\tau_{g 1 \|}^{\prime}= \pm \xi_{\|} \frac{28}{25} \frac{g_{0} P_{P} L_{e f f}}{2 \pi A_{e f f}} \frac{1}{\left(\frac{\Delta v}{2}\right)}
$$

In a similar way, maximum/minimum group delay along the $y$ axis at the same frequencies can be obtained 

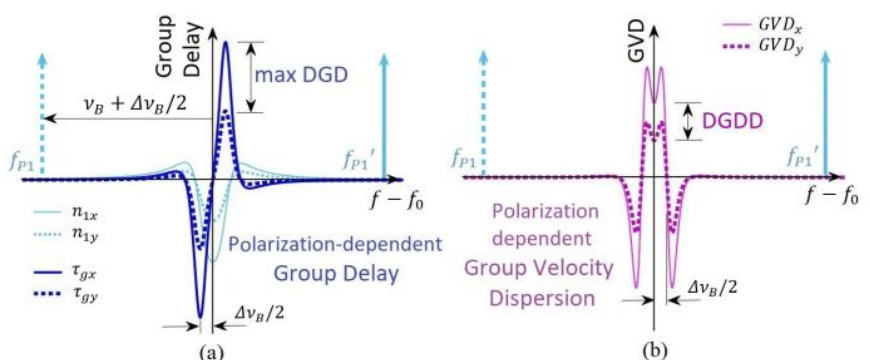

Fig. 5. SBS-induced (a) group delay and (b) group velocity dispersion responses of two pumps symmetrically placed at $f_{0} \mp\left(v_{B}+\Delta v_{B} / 2\right)$. Effects produced along $x$ and $y$ axis are plot in solid and dashed lines, respectively.

$1 \tau_{g y-\max / \min }=\tau_{g 1 \perp}+\tau_{g 1 \perp}^{\prime}= \pm \xi_{\perp} \frac{28}{25} \frac{g_{0} P_{P} L_{e f f}}{2 \pi A_{e f f}} \frac{1}{\left(\frac{\Delta v}{2}\right)}$.

A graphical representation of both delays can be seen in Fig. 5a. By employing (12), the maximum induced DGD can be determined

$$
\mathrm{DGD}_{\max }=\delta \xi \frac{1}{2 \pi} \frac{28}{25} \frac{g_{0} P_{P} L_{e f f}}{A_{\text {eff }}} \frac{1}{\left(\frac{\Delta v}{2}\right)}
$$

For frequencies $f<f_{0}$, the $y$ axis takes the role of fast axis 45 since its induced group delay is positive. Meanwhile, if a light 6 beam with frequencies $f>f_{0}$ is set in the $x$ polarization axis, it7 will propagate faster than if it would be in the $y$ polarization 48 axis. In other words, $x$ and $y$ axis act as a frequency dependent9 fast/slow light generator pair. Thus, DGD can be easily $y_{0}$ manipulated by adjusting the power of pumps in the $x$ and $\$ 1$ axis. However, the SBS-induced gain/loss response (Fig. 1.a\$2 must also be considered. It affects the signal at $f_{0} \pm \Delta v_{B} / 2$ by 3 intrinsically generating a single sideband modulation $_{54}$ Therefore, using only a single pair of pumps it is expected t95 experience only slow light signals.

\section{SBS-induced Dynamic Differential Group Delay Dispersion}

Another property derived from the DGD is the DGE $^{9}$ dispersion (DGDD) or second-order DGD. This is determine 60 as the difference between slopes of the induced group delays o 1 $x$ and $y$ axis. Each slope is, by definition, the induced grou 62 velocity dispersion (GVD) in the corresponding propagatio 33 axis. At $f_{0}$, it is

$$
\begin{aligned}
\mathrm{DGDD} & =\mathrm{GVD}_{x}-\mathrm{GVD}_{y} \\
& =\delta \xi \frac{1}{(2 \pi)^{2}} \frac{g_{0} P_{p} L_{e f f}}{A_{e f f} L} \frac{1}{(\Delta v / 2)^{\wedge} 2} .
\end{aligned}
$$

As it is shown in Fig. 5b, this value is not uniform over the 70 bandwidth $\Delta v$ and is not the maximum value. As in previous $7_{2}$ studied cases, DGDD can be controlled by modifying the pump $7_{3}$ power.

Analogous analysis can be carried out for the secondary pairs 75 of pumps $f_{P 2}$ and $f_{P 2}{ }^{\prime}$, resulting in the curves of Fig. 5 but 76 inverted. Additionally, the transformation enounced in Section IIA for a different pair of orthogonal pumps is also valid.

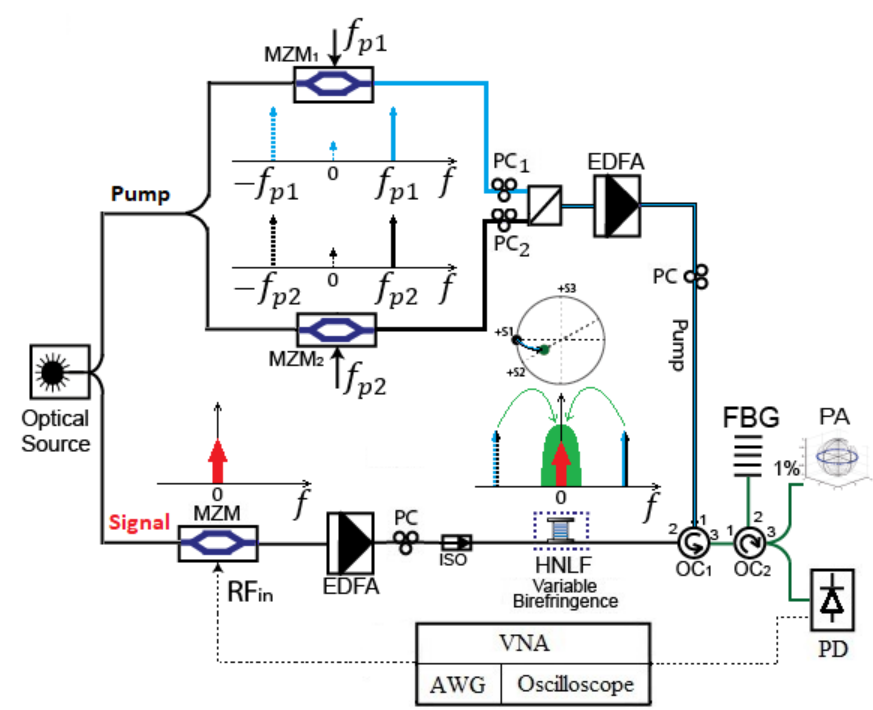

Fig. 6. Experimental setup.

\section{EXPERIMENTAL SETUP}

Experiments have been carried out to proof the concept of an arbitrary birefringent element, differential group delay and differential group delay dispersion, induced by SBS.

Figure 6 shows the block diagram used to demonstrate the feasibility of the nonlinearly-induced arbitrary birefringence and its properties.

An optical signal at $\lambda=1548.25 \mathrm{~nm}$ is split into two paths: the upper one is used to generate the pump waves whereas the lower one experiences variable birefringence. The pump is generated by amplitude modulation of the optical signal using two Mach-Zehnder modulators $\left(\mathrm{MZM}_{1}\right.$ and $\left.\mathrm{MZM}_{2}\right)$ at minimum transmission bias (MITB) and fed by two microwave oscillators with frequencies $f_{P 1}$ and $f_{P 2}$. An optical circulator $\left(\mathrm{OC}_{1}\right)$ directs the pump towards an optical fiber where its induced birefringence is controlled. The fiber is made of 1-km highly nonlinear fiber (HNLF). Brillouin parameters for this fiber are $\Delta v_{B}=70 \mathrm{MHz}, g_{0}=7.19 \times 10^{-12} \mathrm{~m} / \mathrm{W}, A_{\text {eff }}=11$ $\mu \mathrm{m}^{2}$ and $v_{B}=9.64 \mathrm{GHz}$ at $1548 \mathrm{~nm}$. A fiber Bragg grating (FBG) in reflection mode (bandwidth of $12.5 \mathrm{GHz}$ ) is used to filter out backward residual pump waves. Birefringence is controlled by adjusting the pump power through the EDFA and changing the SOP of the pump waves.

In experiments related to DGD, the SOP of the primary pair of pumps, $f_{P 1}$ and $f_{P 1}{ }^{\prime}$, is set to horizontal linear polarization and the SOP of the secondary pair of pumps, $f_{P 2}$ and $f_{P 2}{ }^{\prime}$, in vertical linear. In the signal path, a Mach-Zehnder modulator (MZM) at quadrature bias point fed by an arbitrary waveform generator (AWG, Rigol DG1062) is used to generate optical pulses. To induce DGD by SBS, the signal is set in horizontal SOP by a polarization controller (PC) and the primary pump is turned on. Then, the primary pump is turned off and the secondary pump is enabled. A photodiode (PD) receives the optical signal and converts it to the electrical domain. Finally, the output signal is analyzed by a Vector Network Analyzer (VNA, HP8510C) and its waveform is acquired by digital oscilloscope (Tektronix TDS 3032) to be further processed. 


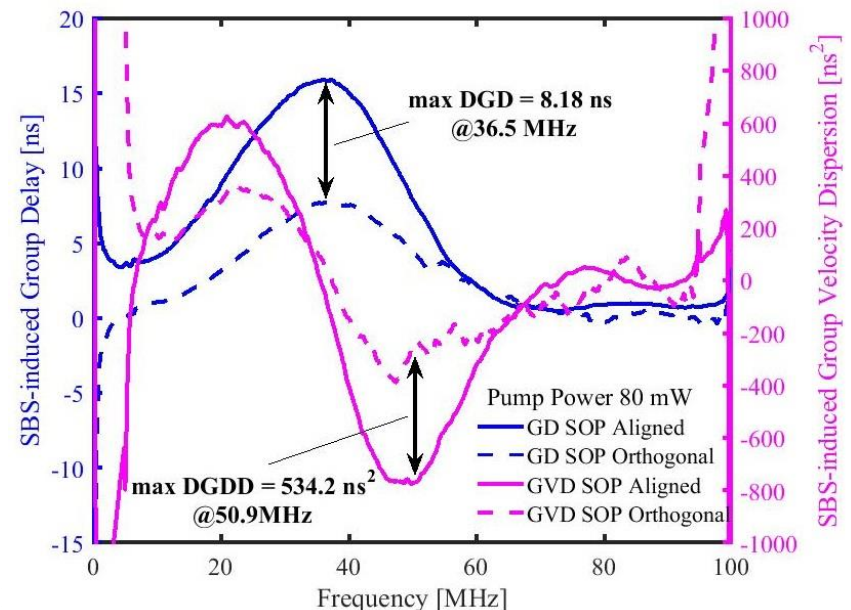

Fig. 10. SBS-induced group delay (blue) and group velocity dispersion (magenta) frequency responses of a transmitted signal with SOP aligned (solid line) and orthogonal (dashed line) to the pump SOP.

Thermal variations will change $v_{B}$, and, in consequence, it will be necessary to readjust pumps' frequencies. Vibrations also affect the states of polarization of pump and signal, which is reflected as additional noise in the signal. Despite this, no significant changes in the system behavior have been observed during the experiments.

\section{A. SBS-induced Dynamic Arbitrary Birefringence}

The Brillouin-induced birefringence grows with Brillouing gain, which in turn is proportional to the effective length and pump power. This dependence can be seen in Fig. 7, where birefringence has been estimated from the retardance measure using the Poincaré sphere method [15]. It shows measurements of the SBS-induced birefringence for an input signal of 0.0 $\mathrm{mW}$ as a function of pump power for three types of birefringence: linear $\left([(1,0)]^{T},[(0,1)]^{T}\right)$, circular $\left(\left[1 / \sqrt{2}\left(1_{35}\right.\right.\right.$ $\left.j)]^{T},[1 / \sqrt{2}(1,-j)]^{T}\right)$ and elliptical $\left([1 / \sqrt{2}(1+j, 0)]^{T},([1 \not 66\right.$ $\left.\sqrt{2}(0,1-j)]^{T}\right)$. Figure 7 also shows that this technique caв 7 provide different kinds of birefringence with the sam88 efficiency, i.e., the same pump power induces the sam89 retardance for linear, circular, and elliptical birefringence. In aly0 cases, the relation is linear. The magnitude of the birefringenc 1

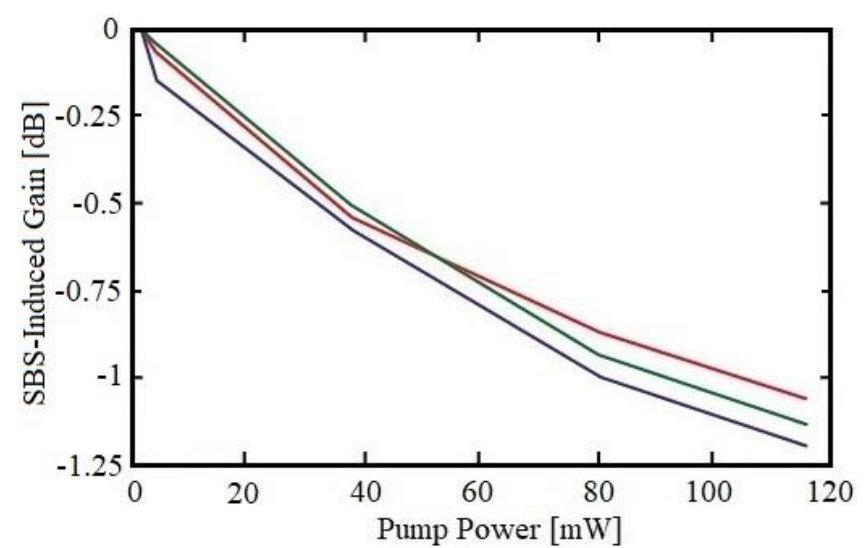

12

Fig. 8. SBS-induced gain at the central frequency as a function of pump power ${ }^{j 4}$ for (blue) linear, (red) circular and (green) elliptical induced birefringence. i5

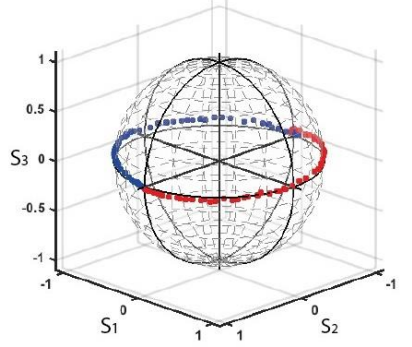

(a)

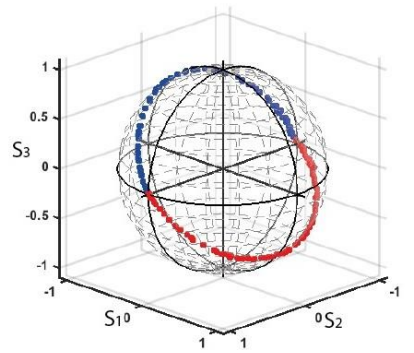

(c)

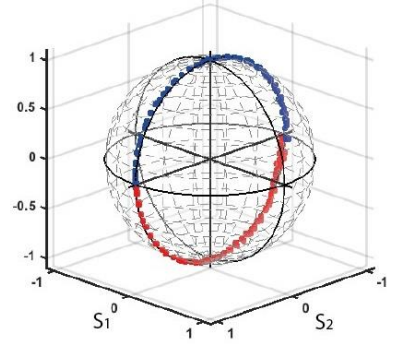

(b)

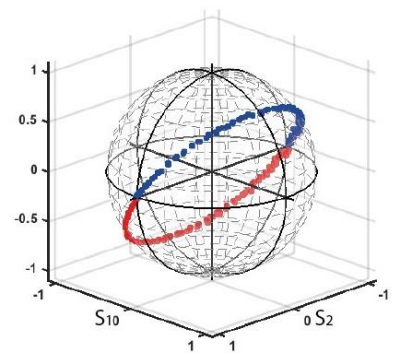

(d)
Fig. 9. Evolution of the state of polarization represented on the Poincare sphere for different types of nonlinearly induced birefringence: a) circular, b) linear; c) and d) elliptical.

can be continuously tuned by changing pump power.

When a change in the birefringence is performed, only a slight variation in the SBS-induced gain is observed because of the combination of gain and loss responses, as shown in Fig. 8. Results show that the SBS-induced gain depends on the pump power and the type of induced birefringence, remaining below $1.1 \mathrm{~dB}$ in the full range of operation.

Figure 9a shows the evolution of the signal SOP at the output of the optical fiber when circular birefringence has been nonlinearly induced. It shows that when the power of the pump is changed from $5 \mathrm{~mW}$ to $90 \mathrm{~mW}$ (Fig. 7) half of a circumference (blue dots) on the Poincaré sphere is described. The rest of the circumference is covered by swapping the pumps $f_{P 1}$ and $f_{P 2}$ (red dots), so in this way it is avoided an excessive increment of the pump power, which could compromise system linearity.

For the linear birefringence, the rotation is obtained in the same way, but the SOPs of the pump waves are linear at $90^{\circ}$ and $0^{\circ}$ (Fig. 9b). Finally, Fig. 9c and Fig. 9d show elliptical birefringence when the SOPs of the pump waves are elliptical with Jones vectors $\left([1 / \sqrt{2}(1+j, 0)]^{T},\left([1 / \sqrt{2}(0,1-j)]^{T}\right)\right.$ and $\left([1 / \sqrt{2}(0,1+j)]^{T},\left([1 / \sqrt{2}(1-j, 0)]^{T}\right)\right.$, respectively.

\section{B. SBS-induced Dynamic Differential Group Delay and Differential Group Delay Dispersion}

Figure 10 presents the results obtained for the SBS-induced group delay (blue) and group velocity dispersion (magenta). Again, the polarization-dependent characteristics of both responses can be appreciated. Maximum group delay is reached at $36.5 \mathrm{MHz}$ for both exposed cases (when the signal SOP is aligned to the pump SOP and when it is orthogonal to the pump SOP). From this difference the maximum differential group delay (DGD), which is $8.18 \mathrm{~ns}$ in a $1 \mathrm{~km}$ fiber reel using a pump power of $80 \mathrm{~mW}$, can be extracted. For the sake of comparison, 
1 the PMD parameter value, $\mathrm{D}_{\mathrm{PMD}}$, associated to this SBS2 induced DGD is around 16000 times higher than the RMS value 3 of DGD produced by the propagation of a light beam through 4 the same distance of standard single mode fiber, typically 5 around $\mathrm{D}_{\mathrm{PMD}}=5 \mathrm{ps} \mathrm{km}^{-1 / 2}$.

Group velocity dispersion (magenta), determined by the derivative of group delay responses, takes positive and negative values around $36.5 \mathrm{MHz}$, at the point where group delay reaches 9 a peak. The maximum negative value of GVD is $-800 \mathrm{~ns}^{2}$ at 45 $10 \mathrm{MHz}$, when the signal SOP is aligned to the pump SOP. By considering that the length of the employed fiber is $1 \mathrm{~km}$, the resultant GVD value is much greater than the typical value of GVD parameter in a standard single mode fiber $\left(\beta_{2}=20\right.$ $\mathrm{ps}^{2} / \mathrm{km}$ ). When signal and pump SOPs are orthogonal, the GVD is reduced a $50 \%$. Located at the frequency of $50.9 \mathrm{MHz}$, the maximum measured DGDD value is $534.2 \mathrm{~ns}^{2}$.

Additional tests were carried out using pulses with a full width at half maximum (FWHM) of $50 \mathrm{~ns}$ modulated in amplitude at a frequency of $35 \mathrm{MHz}$ (in the region of maximum DGD as shown in Fig. 10), which were transmitted into the arbitrary birefringent system to test the DGD. As other SBSbased systems, the limited time-bandwidth product prevents the possibility of delaying narrower pulses [10]. The results are shown in Fig. 11. When the pump is on and its SOP is aligned to the signal, the signal experiences a stronger delay (red solid line) in relation to the signal without pump (black solid line) and also to the signal orthogonal to the pump SOP (blue dashed line). The measured DGD was $7 \mathrm{~ns}$ using $100 \mathrm{~mW}$ of pump power. As discussed in Section II.B, the sign of the group delay cannot be changed since it can only be induced using the gain response of the SBS. Unlike baseband signals, being away from $f_{0}$, the signal amplitude is altered by the SBS gain response, which increases with pump power. Aligned signal exhibits a 8 higher amplitude than the orthogonal due to the polarizatio 9 dependent characteristics of SBS gain response [5][9]. Th $₫ 0$ influence of pump power and its SOP in baseband optical pulses 1 can be seen in Fig. 12. These pulses were demodulated b\$2

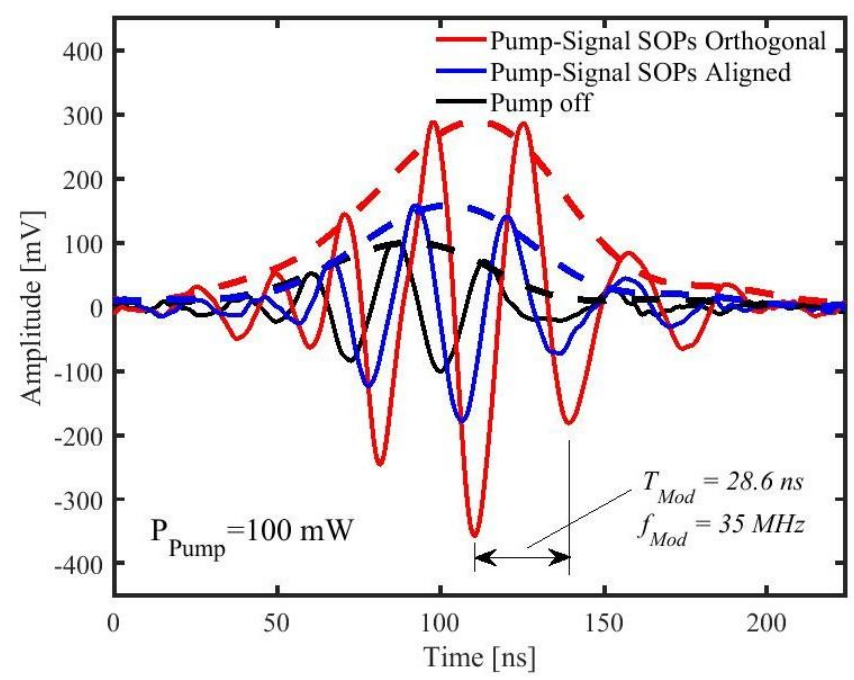

Fig. 11. Measurement of modulated pulses of FWHM $=50 \mathrm{~ns}$ at $35 \mathrm{MHz}$ transmitted through the optical fiber without pump (solid black line) and with SOP aligned (solid red line) and SOP orthogonal (solid blue line) to the pump SOP. Received baseband pulses are represented by the envelope (dashed lines) of the signals.

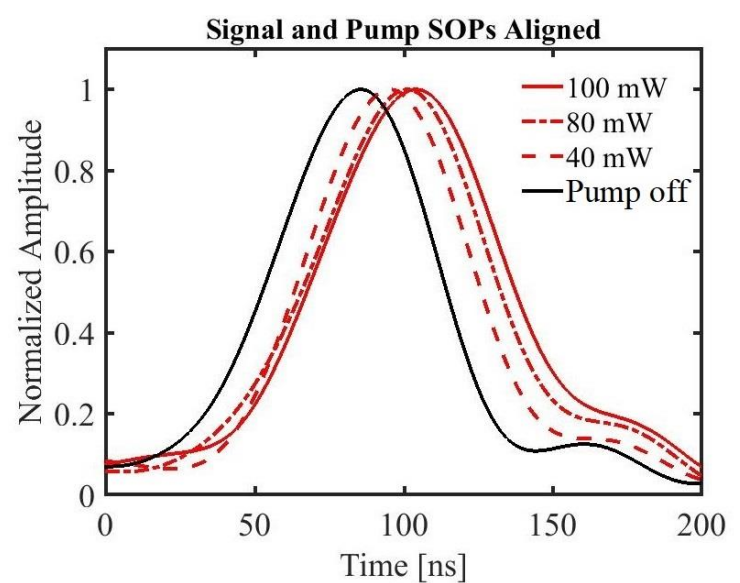

(a)

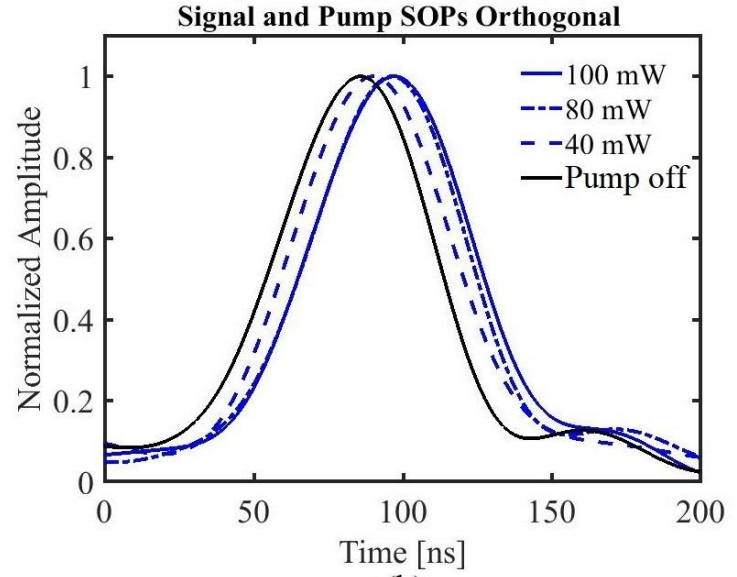

(b)

Fig. 12. Baseband optical pulses (FWHM $=50 \mathrm{~ns}$ ) normalized in peak amplitude affected by SBS when signal and pump SOPs are (a) aligned and (b) orthogonal.

employing digital envelope detection. In the range of pump powers from $10 \mathrm{~mW}$ to $100 \mathrm{~mW}$, distortion in pulses is moderate.

Figure 13 shows the measured group delay of baseband pulses for different pump powers. The DGD is larger as the pump power increases. The maximum group delay obtained was $18.4 \mathrm{~ns}$ using a pump power of $100 \mathrm{~mW}$ when signal and

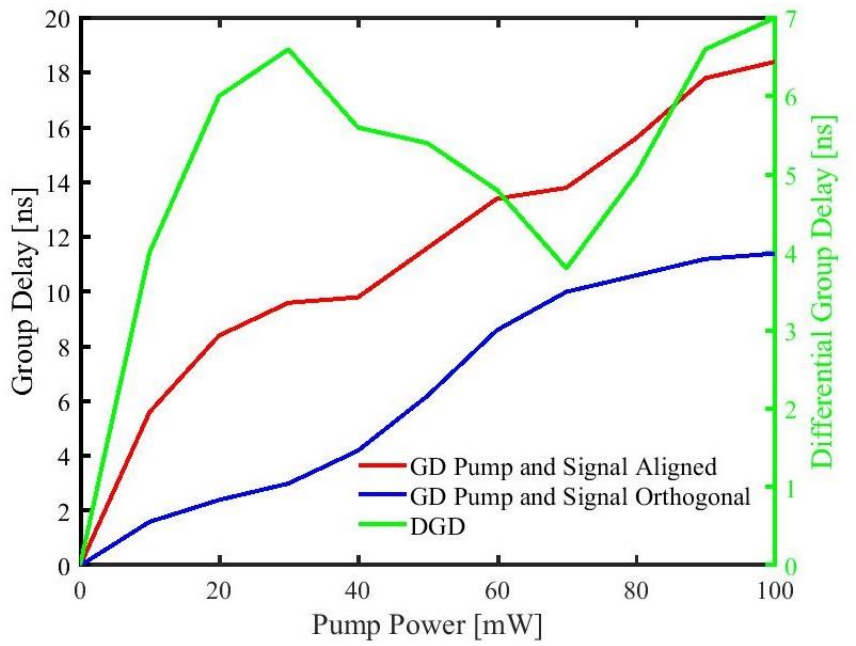

Fig. 13. Group delay (blue and red) and differential group delay (green) as a function of pump power for baseband optical pulses of FWHM = 50 ns affected by SBS. 
1 pump SOPs are aligned. When both are orthogonal, the group8 2 delay was $11.4 \mathrm{~ns}$. The obtained delays are in line with previou39 3 results [9-12].

The system behavior can be alternatively analyzed throug\#1 its frequency response. The SBS-induced gain and phas $\$ 2$ 6 frequency responses have been measured using a vecto 3 network analyzer and the results are shown in Fig. 14. Thæ4 8 polarization dependence of SBS-induced gain and phas 45 9 frequency responses is clearly shown. When the signal SOP $i 46$

10 aligned to the pump SOP (green line), gain and phase response 7 11 are enhanced, with a gain peak of nearly $2 \mathrm{~dB}$ and a phase shift8 of around -140 degrees at $40 \mathrm{MHz}$. On the other hand, th 49 orthogonal case provides a modest maximum gain of less than $1 \mathrm{~dB}$ at $30 \mathrm{MHz}$ and a minimum phase of -40 degrees at 550 MHz. In the range of $20 \mathrm{MHz}$ to $45 \mathrm{MHz}$, phase responses 1 present a well-defined slope. The difference between the slopes 2 of both phase responses indicates the presence of DGD, whic 3 is consistent with the results depicted in Fig. 10.

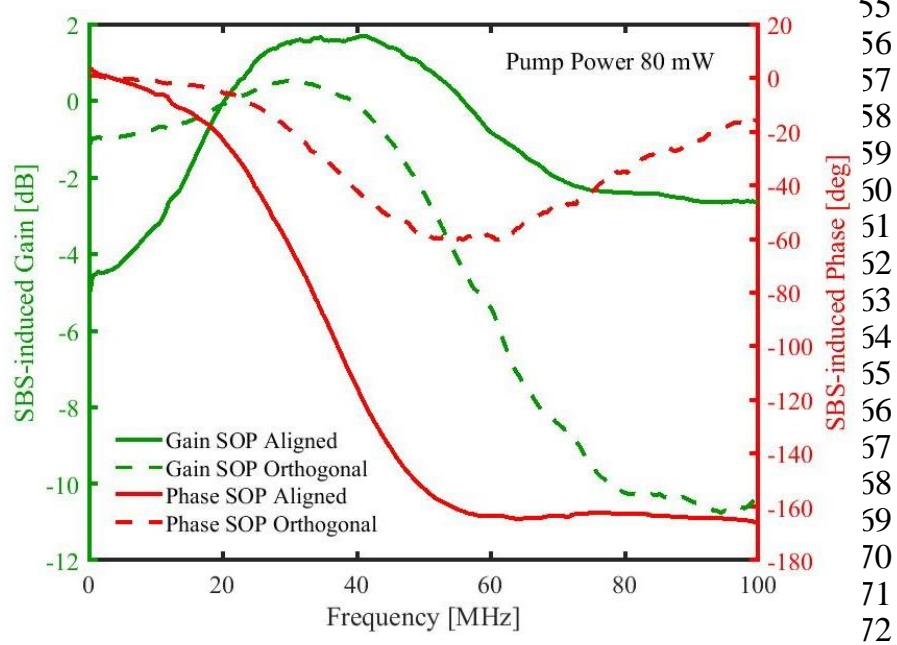

Fig. 14. Frequency responses of the SBS-induced gain (green) and phase (red) 73 of a transmitted signal with SOP aligned (solid line) and orthogonal (dashed 74 line) to the pump SOP. birefringent, delay-generator and highly dispersive propagating media.

Power consumption could be improved by using a more efficient nonlinear medium, such as photonic crystal fibers. This will also induce a larger birefringence, and, in turn, it would allow the simplification of the setup by removing one pump pair.

Further work is needed to simplify the setup, investigate the use of this new approach in optical integrated waveguides exploiting Brillouin effect [15] and extend the concept to other nonlinear effects and thus overcome some limitations of SBS as its limited time-bandwidth product.

\section{REFERENCES}

[1] Y.J. Tsai, S. Larouche, T. Tyler, A. Llopis, M. Royal, N.M. Jokerst, D.R. Smith, "Arbitrary birefringent metamaterials for holographic optics at $\lambda=$ 1.55 um," Opt. Express, vol. 21, no. 22, pp. 26620-26630, Nov. 2013.

[2] A. Cerjan, S. Fan, "Achieving arbitrary control over pairs of polarization states using complex birefringent metamaterials," Phys. Rev. Lett., 118, 253902, May 2011.

[3] A. J. Danner, T. Tyc, U. Leonhardt, "Controlling birefringence in dielectrics," Nature Photon., 5, pp. 357-359, Nov. 2013.

[4] A. Bergman, M. Tur, "Brillouin Dynamic Gratings - A Practical Form of Brillouin Enhanced Four Wave Mixing in Waveguides: The First Decade and Beyond", Sensors, vol. 18, no. 9, pp. 2863, 2018.

[5] A. Zadok, E. Zilka, A. Eyal, L. Thevenaz, M Tur, "Vector analysis of stimulated Brillouin scattering amplification in standard single-mode fibers," Opt. Express., vol. 16, no. 26, pp. 21692-21707, Dec. 2008.

[6] Z. Schmilovitch, N. Primerov, A. Zadok, A. Eyal, S. Chin, L. Thevenaz, M. Tur, "Dual-pump push-pull polarization control using stimulated Brillouin scattering," Opt. Exp., vol. 19, no. 27, pp. 25873, Dec. 2011.

[7] D. Samaniego, B. Vidal, "Brillouin wavelength selective all-optical polarization conversion," Phot..Res., vol. 8, no. 4, pp. 440-447, Apr. 2020.

[8] M. Gonzalez-Herráez, K.Y. Song, L. Thevenaz, "Arbitrary-bandwidth Brillouin slow light in optical fibers," Opt. Express, vol. 14, no. 4, pp. 1395-1400, Feb. 2006.

[9] T. Schneider, R. Henker, K.U. Lauterbach, M. Junker, "Comparison of delay enhancement mechanisms for SBS-based slow light systems," Opt. Express, vol. 15, no. 15, pp. 9606-9613, Jul. 2007.

[10] S. Chin, L. Thévenaz, "Tunable photonic delay lines in optical fibers," Laser \& Photon. Review, 6, no.6, pp. 724-738, Feb. 2012.

[11] Y. Okawachi, M.S. Bigelow, J.E. Sharping, Z. Zhu, A. Schweinsberg, D.J. Gauthier, R.W. Boyd, A.L. Gaeta, "Tunable All-Optical Delays via Brillouin Slow Light in Optical Fiber”, Phys. Rev. Lett. 94, 153902, 2005.

[12] A. Choudhary, Y. Liu, B. Morrison, H. Vu, D.Y. Choi, P. Ma, S. Madden, D. Marpaung, B.J. Eggleton, "High-resolution, on-chip RF photonic signal processor using Brillouin gain shaping and RF interference," Sci. Rep., vol. 7, pp. 5932, 2017.

[13] A. Zadok, S. Chin, L. Thévenaz, E. Zilka, A. Eyal, M. Tur, "Polarizationinduced distortion in stimulated Brillouin scattering slow-light systems", Optics Letters, vol. 34, no. 16, pp. 2530-2532, August 2009.

[14] D. Derickson, Fiber Optic Test and Measurement, Ed. Prentice Hall, 1998.

[15] B.J. Eggleton, C.G. Poulton, P.T. Rakitch, M.J. Steel, G. Bahl, "Brillouin integrated photonics," Nat. Photonics, vol. 13, pp. 664-677, Oct. 2019. 